\title{
Ices in Star-Forming Regions: First Results from VLT-ISAAC
}

Ewine F. van Dishoeck ${ }^{1,4}$, E. Dartois ${ }^{2}$, W.F. Thi ${ }^{1}$, L. d'Hendecourt $^{2}$, A.G.G.M. Tielens ${ }^{3}$, P. Ehrenfreund ${ }^{4}$, W.A. Schutte ${ }^{4}$, K. Pontoppidan ${ }^{1}$, K. Demyk $^{2}$, J. Keane ${ }^{3}$, and A.C.A. Boogert ${ }^{5}$

1 Leiden Observatory, P.O. Box 9513, NL-2300 RA Leiden, The Netherlands

2 'Astrochimie Expérimentale', Université Paris XI, Bat. 121, F-91405 Orsay, France

3 Kapteyn Institute/SRON Groningen, P.O. Box 800, NL-9700 AV Groningen, The Netherlands

4 Raymond \& Beverly Sackler Laboratory for Astrophysics, Leiden Observatory, P.O. Box 9513, NL-2300 RA Leiden, The Netherlands

5 Div. of Physics, Mathematics \& Astronomy, Caltech, Pasadena, USA

\begin{abstract}
The first results from a VLT-ISAAC program on the infrared spectroscopy of deeply-embedded young stellar objects are presented. The advent of 8-m class telescopes allows high $S / N$ spectra of low-luminosity sources to be obtained. In our first observing run, low- and medium-resolution spectra have been measured toward a dozen objects, mostly in the Vela and Chamaeleon molecular clouds. The spectra show strong absorption of $\mathrm{H}_{2} \mathrm{O}$ and $\mathrm{CO}$ ice, as well as weak features at ' 3.47 ' and $4.62 \mu \mathrm{m}$. No significant solid $\mathrm{CH}_{3} \mathrm{OH}$ feature at $3.54 \mu \mathrm{m}$ is found, indicating that the $\mathrm{CH}_{3} \mathrm{OH} / \mathrm{H}_{2} \mathrm{O}$ ice abundance is lower than toward some massive protostars. Various evolutionary diagnostics are investigated for a set of sources in Vela.
\end{abstract}

\section{Introduction}

Interstellar matter provides the basic building blocks from which new solar systems like our own are made. The formation of stars and planetary systems begins with the collapse of a dense interstellar cloud core, a reservoir of dust and gas from which the protostar and circumstellar disk are assembled. In this cold and dense phase, molecules freeze-out onto the grains and form an icy mantle surrounding the silicate and carbonaceous cores. The ices can contain up to $40 \%$ of the condensible material (i.e., C and O) (Whittet et al. 1998, d'Hendecourt et al. 1999). Much of this material is incorporated into the circumstellar disks and ultimately in icy solar system bodies such as comets (Ehrenfreund et al. 1997). A central quest in star formation and astrochemistry is to understand the evolution of these species from interstellar clouds to planetary bodies, and use them as diagnostic probes of the thermal history and physical processes (van Dishoeck \& Blake 1998, Ehrenfreund \& Charnley 2000, Langer et al. 2000).

In recent years, much progress has been made in our understanding of the chemical evolution during star formation through combined submillimeter and infrared observations. Submillimeter observations using telescopes such as the JCMT, CSO, IRAM 30m and SEST probe the gas-phase composition of the warm and dense envelopes around deeply-embedded protostars. Many different 
species with abundances down to $10^{-11}$ with respect to $\mathrm{H}_{2}$ can be probed through their pure rotational transitions. Because of the high spectral resolution of the heterodyne technique $\left(R=\lambda / \Delta \lambda>10^{6}\right)$, the line profiles are resolved and provide information on the location of the molecules (e.g., quiescent gas vs. outflow). Since the lines are in emission, a map of their distribution can be made.

Infrared spectroscopy provides complementary information: at these wavelengths the vibrational modes of both gas-phase and solid-state species can be observed, but only down to abundances of $\sim 10^{-7}$ with respect to $\mathrm{H}_{2}$ at typical resolving powers $R \approx$ a few thousand. Symmetric molecules, such as $\mathrm{H}_{3}^{+}, \mathrm{CH}_{4}$, $\mathrm{C}_{2} \mathrm{H}_{2}$ and $\mathrm{CO}_{2}$ have no dipole-allowed rotational transitions, and can therefore only be probed through their strong infrared vibrational transitions. Moreover, $\mathrm{PAH}$ emission features appear throughout the mid-infrared range, and the dominant interstellar molecule, $\mathrm{H}_{2}$, has its pure rotational transitions at these wavelengths. The cold gases and ices are usually observed in absorption toward an embedded YSO, where the hot dust in the immediate circumstellar environment provides the continuum. This technique samples only a pencil beam line of sight. The Short Wavelength Spectrometer (SWS) on the Infrared Space Observatory (ISO) has opened up the mid-infrared wavelength region and has obtained spectra of more than a dozen protostars without atmospheric interference (see van Dishoeck \& Tielens 2001 for review). However, ISO was limited to the most luminous, massive YSOs $\left(L \approx 10^{4}-10^{5} \mathrm{~L}_{\odot}\right)$. A major goal of our VLT-ISAAC program is to extend this work to lower-mass objects $\left(L \leq 10^{3} \mathrm{~L}_{\odot}\right)$ representative of our proto-Sun.

Together, the submillimeter and infrared data have led to the following scenario for high-mass objects. In the cold pre-stellar cores and collapsing envelopes, gas-phase molecules freeze-out onto the grains and form an icy mantle. Here the abundances can be further modified by grain surface reactions and, perhaps, photoprocessing of ices. In particular, the hydrogenation and oxidation of accreted $\mathrm{C}, \mathrm{O}, \mathrm{N}$ and $\mathrm{CO}$ can lead to $\mathrm{CH}_{4}, \mathrm{H}_{2} \mathrm{O}, \mathrm{NH}_{3}, \mathrm{H}_{2} \mathrm{CO}, \mathrm{CH}_{3} \mathrm{OH}$ and $\mathrm{CO}_{2}$, respectively (Tielens \& Charnley 1997). Most of these species have been firmly identified in interstellar ices (e.g., Whittet et al. 1996, d'Hendecourt et al. 1996, Gibb et al. 2000). Once the protostar has formed, its luminosity can heat the surrounding grains to temperatures at which the ices evaporate back into the gas phase, resulting in enhanced gas/solid ratios (e.g., van Dishoeck et al. 1996, Dartois et al. 1998, Boonman et al. 2000). The sublimation temperatures range from $\sim 20 \mathrm{~K}$ for pure $\mathrm{CO}$ ice to $\sim 90 \mathrm{~K}$ for $\mathrm{H}_{2} \mathrm{O}$-rich ice under typical conditions. These freshly evaporated molecules can then drive a rich and complex chemistry in the gas (called the 'hot core' phase) until the normal ion-molecule chemistry takes over again after $\sim 10^{5}$ yr (Charnley et al. 1992). The different solid-state and gas-phase species therefore serve not only as physical diagnostics, but also as probes of the evolution of the region. 


\section{Our VLT-ISAAC program}

In 1999, we proposed a large VLT-UT1 ISAAC program to probe the origin and evolution of ices in southern star-forming regions through a spectroscopic survey of $30-40$ objects in the 2.7-5.1 $\mu \mathrm{m} \mathrm{L}$ and M-band atmospheric windows. The aim was to obtain high-quality spectra $(S / N>50$ on continuum) such that $3 \sigma$ limits of species with abundances down to $2-4 \%$ of $\mathrm{H}_{2} \mathrm{O}$ ice - the dominant ice component - can be obtained. The program focuses on low- and intermediatemass southern YSOs, but covers a range of evolutionary stages from background stars to T-Tauri stars with disks. Also, several different environments (Vela, Chamaeleon, Ophiuchus, Corona Australis, ...) will be probed. The program was awarded 14 nights of VLT time. However, due to technical problems with the long-wavelength arm of ISAAC, the first observing session did not take place until January 2001.

The January 2001 observations totalled 5 nights and were carried out under mediocre conditions with high humidity. Nevertheless, good low-resolution (LR, $R=600-800)$ spectra were obtained for $\sim 15$ objects and medium-resolution (MR, $R=3000-5000)$ spectra for $\sim 5$ objects, covering $\sim$ one-third of our project. With the new $1024 \times 1024$ Aladdin array, the low-resolution spectra can be obtained in a single spectral setting per atmospheric window, whereas the medium resolution spectra require $6 / 3$ settings to cover the entire $\mathrm{L} / \mathrm{M}$ band, respectively. Typical exposure times are $\sim 30$ minutes per atmospheric window in $\mathrm{LR}$ for a $\mathrm{L} \approx 7$ mag object. Standard stars were observed immediately before or after the YSOs, within 0.05-0.1 airmass. The spectra were obtained using both chopping (by $15^{\prime \prime}$ along the slit) and nodding of the telescope. Daily arcs and flat-fields were provided by the observatory staff. In the M-band, the atmospheric $\mathrm{CO}$ lines were used for wavelength calibration. The data were reduced using IDL routines developed in-house by E. Dartois and W.F. Thi.

Because the ice features are weak and broad and are superposed on a strong continuum, many tests were carried out to check the reliability of the spectra. Specifically, spectra were taken for objects also observed with ISO or UKIRT, and features were checked on different nights at the VLT and between the LR and MR modes on the VLT. In general, the reproducibility of the features is excellent (see Fig. 1).

A particularly nice capability of ISAAC is the possibility to rotate the slit on the sky to obtain spectra of more than one object simultaneously. In several cases, more than one bright object at L or M-band was discovered in the acquisition image within $\sim 15^{\prime \prime}$. The slit was then rotated to include the additional object. An example is shown in Fig. 2 for the high-mass source GL 961, where the E and $\mathrm{W}$ components are separated by $\sim 5.5^{\prime \prime}$.

Laboratory data such as those obtained in the Sackler Laboratory for Astrophysics in Leiden and at the Astrochimie Expérimentale Laboratory in Paris play an essential role in the analysis of the infrared spectra. First, they lead to definite identification of the molecules and to quantitative estimates of their abundances. Second, they provide an indication of the ice environment and ice components. For example, a $\mathrm{CO}$ molecule embedded in an $\mathrm{H}_{2} \mathrm{O}$-rich matrix ('polar ice') has a 


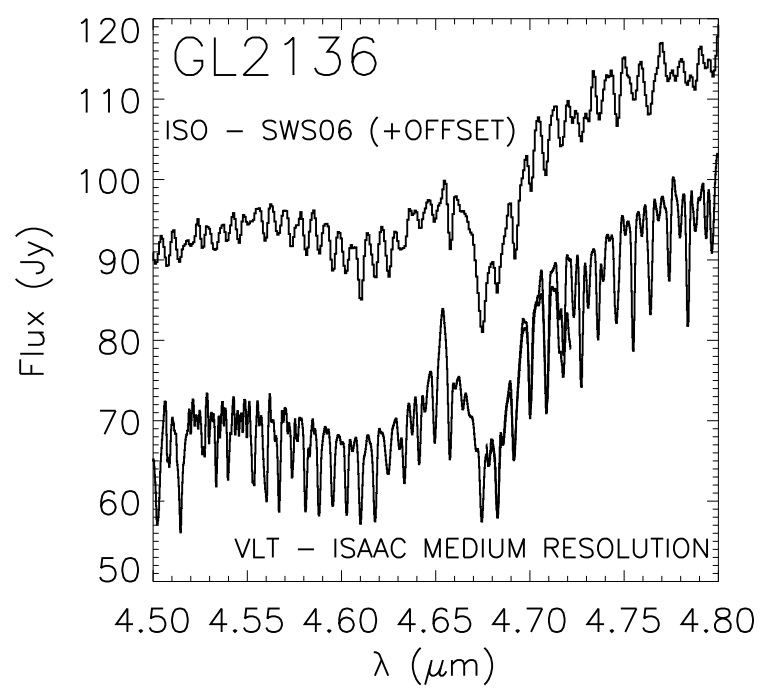

Fig. 1. VLT-ISAAC MR M-band $R \approx 2000$ spectrum of GL 2136 compared with the ISO-SWS spectrum $(R \approx 1500)$. The sharp features are due to gas-phase CO, and become stronger in the higher-resolution VLT spectrum. Broad absorption features at $4.62 \mu \mathrm{m}$ due to solid $\mathrm{OCN}^{-}$and at $4.67 \mu \mathrm{m}$ due to solid $\mathrm{CO}$ are seen as well. The $\mathrm{H}$ I 7-5 emission line at $4.6538 \mu \mathrm{m}$ is stronger in the VLT spectrum due to the smaller slit.

different spectral shape compared with that in a CO-rich mantle ('apolar ice'). Such analyses have shown that the ice mantles in the protostellar environment are not homogeneous, but consist of several components. These phases may reflect differential accretion of atomic H-rich versus H-poor gas and/or different degrees of outgassing of the more volatile species (Schutte 1999). Third, the ice spectra and the gas/solid ratios can provide clear evidence for heating of the ices in the more evolved objects (Ehrenfreund et al. 1998, Boogert et al. 2000, van Dishoeck et al. 1996).

\section{$3 \quad$ Initial results}

The prime targets in the January 2001 run were a set of low- and intermediate mass YSOs in the Vela and Chamaeleon clouds, together with a few well-known southern high-mass protostars. In the first case, the power of the VLT+ISAAC is used to observe weaker and lower luminosity sources than possible previously, whereas for the high-mass sources the aim was to obtain higher $S / N$ and higher spectral resolution data than provided by ISO or previous ground-based observations. 

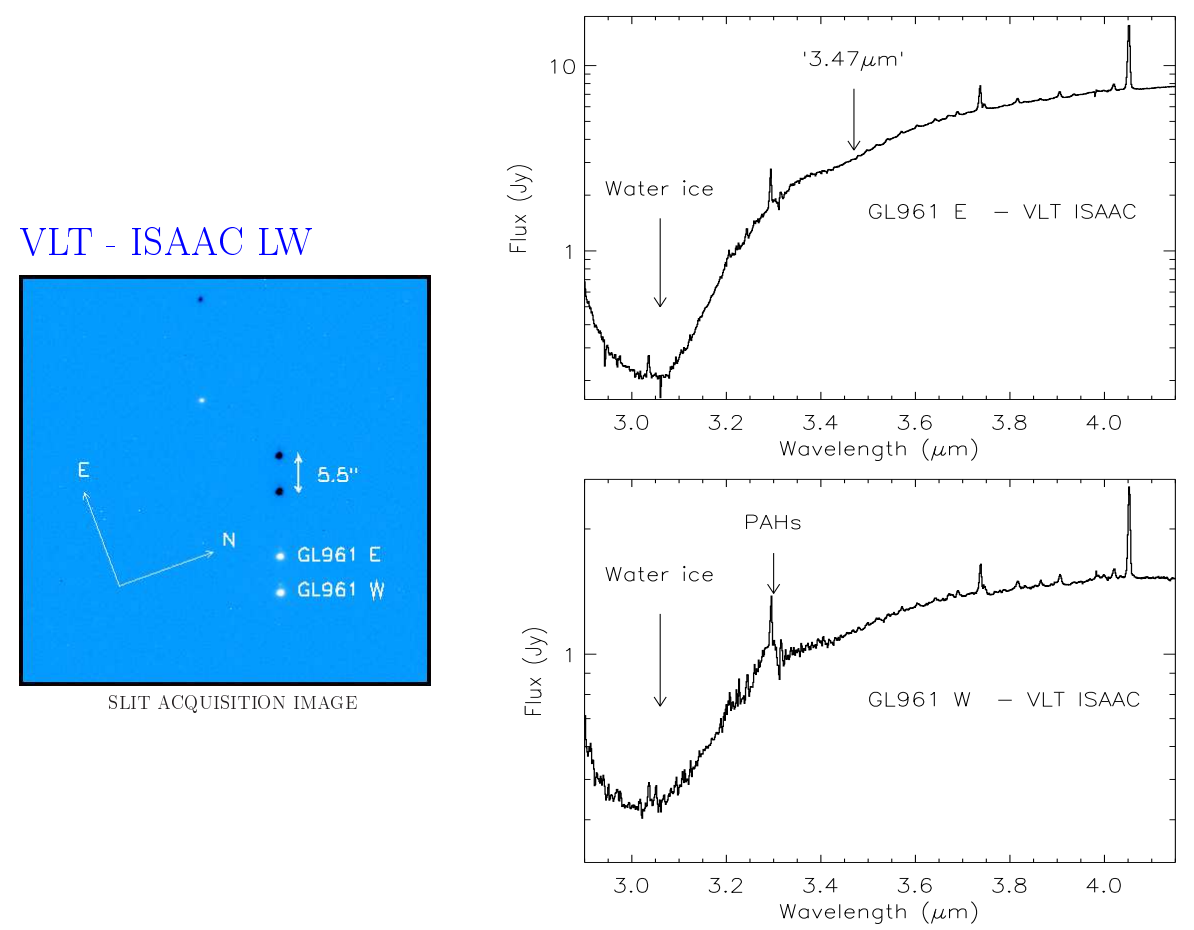

Fig. 2. VLT-ISAAC spectra obtained toward GL $961 \mathrm{E}$ and W. The $3.3 \mu \mathrm{m}$ PAH feature may be affected by poor cancellation of an atmospheric feature.

The L- and M-band windows include the following main features: $3 \mu \mathrm{m}\left(\mathrm{H}_{2} \mathrm{O}\right.$ ice), $3.47 \mu \mathrm{m}$ (unidentified), $3.54 \mu \mathrm{m}\left(\mathrm{CH}_{3} \mathrm{OH}\right.$ ice), $4.08 \mu \mathrm{m}$ (HDO ice), $4.62 \mu \mathrm{m}$ $\left(\mathrm{OCN}^{-}\right.$ice) and $4.67 \mu \mathrm{m}$ (CO gas and ice). The strong solid $\mathrm{H}_{2} \mathrm{O}$ and $\mathrm{CO}$ bands are detected in most objects, although with varying amounts. Typical LR and MR spectra are shown in Figures 1-4. Because of the strong atmospheric features at the relatively low altitude of Paranal, the quality of the data around the solid OCS feature at $4.9 \mu \mathrm{m}$ is low.

Several of these features are excellent indicators of the thermal history and energetic processing of the ices. Specifically, we can use the following diagnostics of the physical conditions and evolution of our sources: (i) the solid $\mathrm{H}_{2} \mathrm{O}$ profile, where the peak position gives an indication of the ice temperature; (ii) the solid $\mathrm{CO}$ profile, where the shape indicates the 'apolar' vs. 'polar' ice fraction; (iii) the $\mathrm{CO} / \mathrm{H}_{2} \mathrm{O}$ abundance ratio, with the more volatile $\mathrm{CO}$ molecule having a lower ice abundance at high temperature; (iv) the gas/solid CO ratio and the gasphase $\mathrm{CO}$ excitation temperature; and (v) the presence of the $\mathrm{OCN}^{-}$feature, which is thought to be a tracer of energetic processing (ultraviolet irradiation or 
particle bombardment) (Schutte \& Greenberg 1997, Demyk et al. 1998). In the following, a few specific initial results are presented.

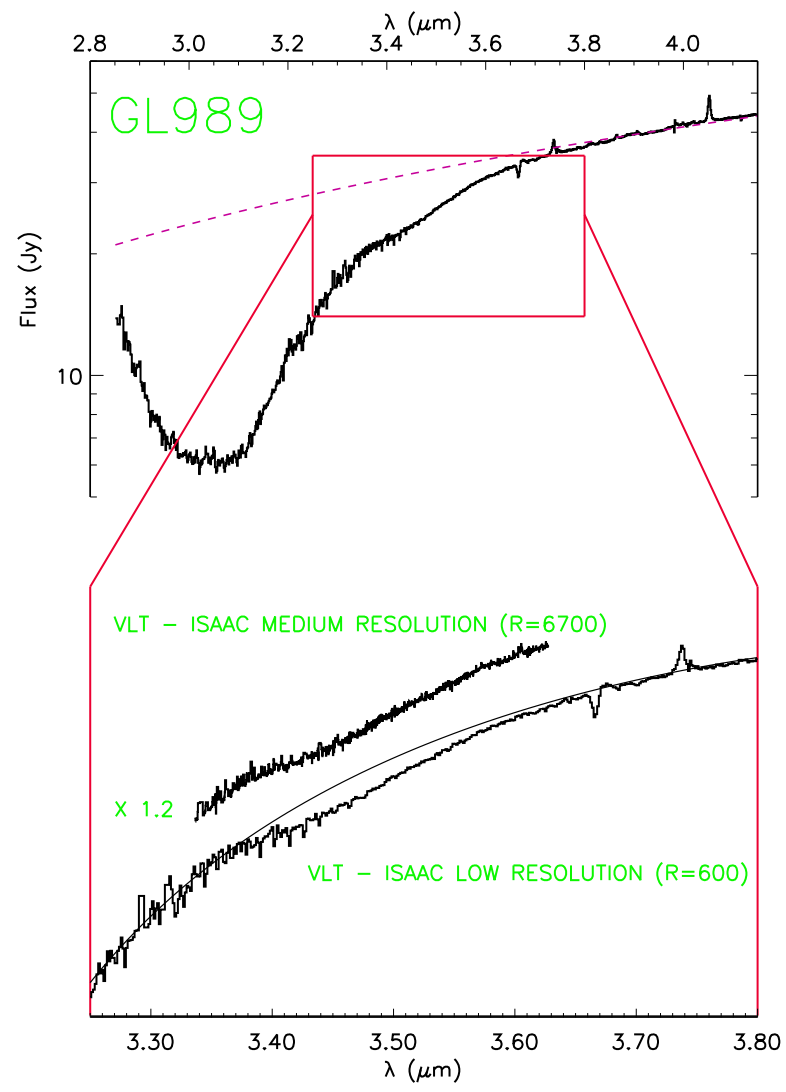

Fig. 3. VLT-ISAAC LR and MR L-band spectra obtained toward GL 989. Note the lack of substructure in the $3.47 \mu \mathrm{m}$ feature (Dartois et al., in prep).

\subsection{The $3.47 \mu \mathrm{m}$ feature}

The $3.47 \mu \mathrm{m}$ feature, previously detected by Allamandola et al. (1992) and Brooke et al. (2000), is observed in several of our sources at high $S / N$. The comparison of the LR and MR spectra for the bright source GL 989 shows good agreement in the shape of the spectra (Figure 3). Also, no substructure is apparent at the higher spectral resolution. The data are currently being compared with different laboratory ice mixtures, in particular mixtures involving $\mathrm{H}_{2} \mathrm{O}$ and $\mathrm{NH}_{3}$ (Dartois et al., in prep.). Theoretical models predict that $\mathrm{NH}_{3}$ is an important component of interstellar ices formed by hydrogenation of atomic $\mathrm{N}$, but unfortunately the strongest $\mathrm{NH}_{3}$ bands are blended with $\mathrm{H}_{2} \mathrm{O}$ at $3 \mu \mathrm{m}$ and with 

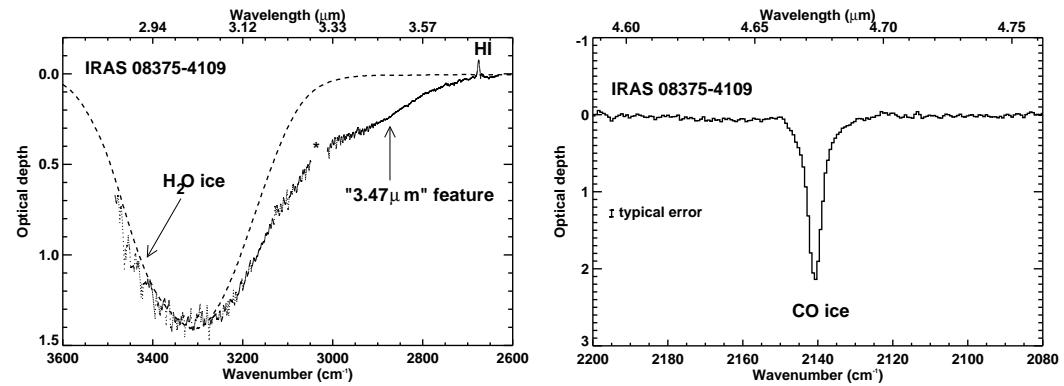

Fig. 4. VLT-ISAAC LR L- and M-band spectra obtained toward IRAS 08375 -4109 in the Vela molecular cloud (Thi et al., in prep).

the silicate band at $9.6 \mu \mathrm{m}$. A tentative detection of the $9.6 \mu \mathrm{m}$ feature toward one (northern) object has recently been claimed by Lacy et al. (1998), suggesting high $\mathrm{NH}_{3}$ abundances up to $10 \%$ with respect to $\mathrm{H}_{2} \mathrm{O}$ ice. The analysis of our VLT data indicates lower $\mathrm{NH}_{3}$ abundances.

\section{$3.2 \quad$ Vela sources}

LR L- and M-band spectra of 5 intermediate mass YSOs $\left(\sim 300-700 \mathrm{~L}_{\odot}\right)$ have been obtained in the previously unexplored Vela molecular cloud (Thi et al., in prep.). The objects were selected from the list of 'class I' objects of Liseau et al. (1992), based on their spectral energy distribution. For two sources, an additional object was found in the field within a few ", providing "off source' information on the ices. MR spectra in the region of the solid and gas-phase CO band have been taken as well for a few cases.

$\mathrm{H}_{2} \mathrm{O}$ ice has been detected in all objects, and $\mathrm{CO}$ ice in half of the objects. The $\mathrm{H}_{2} \mathrm{O}$ profile indicates that the bulk of the ice is very cold. The solid $\mathrm{CO}$ band toward IRAS $08375-4109$ is one of the strongest and sharpest CO bands observed in any source: at LR, the feature is unresolved and its true depth can only be obtained from the MR spectrum (Figure 4). In spite of the low overall temperature, clear differences in the solid $\mathrm{CO} / \mathrm{H}_{2} \mathrm{O}$ ice abundances are observed, which correlate with the bolometric temperature of the source.

Several of the sources show the $3.47 \mu \mathrm{m}$ feature, but the presence of the $3.54 \mu \mathrm{m}$ band is less clear. This limits the solid $\mathrm{CH}_{3} \mathrm{OH}$ abundance to a few $\%$ of that of $\mathrm{H}_{2} \mathrm{O}$ ice, significantly less than the $50 \%$ found toward some massive protostars (Dartois et al. 1999). The $\mathrm{OCN}^{-}$feature is detected in at least one high temperature source.

\subsection{The circumstellar disk around L1489}

L1489 has been shown by Hogerheijde (2001, this volume) to be a transitional object between the class I and II phases. It is surrounded by a large $2000 \mathrm{AU}$ 
E.F. van Dishoeck et al.

radius rotating circumstellar disk, which must be on the verge of shrinking to the $\sim 100$ AU size disks seen around T Tauri stars. Thus, it provides an excellent opportunity to probe the chemical composition of the gas and dust just when it is being incorporated into the disk. The VLT spectra of L1489 show strong absorption by $\mathrm{H}_{2} \mathrm{O}$ and $\mathrm{CO}$ ices, but no evidence for $\mathrm{CH}_{3} \mathrm{OH}$ and $\mathrm{OCN}^{-}$ features, providing limits on their abundances of a few $\%$ with respect to $\mathrm{H}_{2} \mathrm{O}$ ice.

In summary, the initial data show that the VLT-ISAAC is a powerful instrument to obtain high-quality $2.9-5 \mu \mathrm{m}$ spectra of low-luminosity embedded YSOs, and that such data can provide an important step forward in our understanding of the physical and chemical evolution of ices in low-mass young stellar objects and their incorporation into new planetary systems.

\section{References}

1. Allamandola L.J., Sandford S.A, Tielens A.G.G.M., Herbst T.M.: ApJ 399, 134 (1992)

2. Boogert A.C.A. et al: A\&A 353, 349 (2000)

3. Boonman, A. et al.: In ISO beyond the peaks, eds. A. Salama et al., ESA-SP 456, p. $67(2000)$

4. Brooke, T.Y., Sellgren, K., Geballe, T.R.: ApJ 517, 883 (1999)

5. Charnley S.B., Tielens A.G.G.M., Millar T.J.: ApJ 399, L71 (1992)

6. Dartois E., d'Hendecourt L., Boulanger F. et al.: A\&A 331, 651 (1998)

7. Dartois E., Schutte W.A., Geballe T.R. et al.: A\&A 342, L32 (1999)

8. Demyk K., Dartois E., d'Hendecourt L. et al.: A\&A 339, 553 (1998)

9. d'Hendecourt L., Jourdain de Muizon M., Dartois E. et al.: A\&A 315, 365 (1996)

10. d'Hendecourt L., Jourdain de Muizon M., Dartois E. et al.: In The Universe as seen by ISO, ESA-SP (1999)

11. Ehrenfreund, P., Charnley, S.: ARA\&A 38, 427 (2000)

12. Ehrenfreund P., d'Hendecourt L., Dartois E. et al.: Icarus 130, 1 (1997)

13. Ehrenfreund P., Dartois E., Demyk K., d'Hendecourt L.: A\&A 339, L17 (1998)

14. Gibb, E. et al.: ApJ 536, 347 (2000)

15. Hogerheijde M.R.: ApJ 553, 618 (2001)

16. Lacy J.H., Faraji H., Sandford S.A., Allamandola L.J.: ApJ 501, 105 (1998)

17. Langer W.D., van Dishoeck E.F., Blake G.A. et al.: In Protostars 8 Planets $I V$, eds. V. Mannings et al. (Univ. Arizona), p. 29 (2000)

18. Liseau R., Lorenzetti D., Nisini B., Spinoglio L., Moneti A.: A\&A 265, 577 (1992)

19. Schutte W.A. In Solid Interstellar Matter: The ISO Revolution, eds. L. d'Hendecourt, C. Joblin, and A. Jones (EDP, Springer), p. 183 (1999)

20. Schutte W.A., Greenberg J.M.: A\&A 317, L43 (1997)

21. Tielens A.G.G.M., Charnley S.B.: Origin of life and evol. of biosphere 27, 23 (1997)

22. van Dishoeck E.F. et al.: A\&A 315, L349 (1996)

23. van Dishoeck E.F., Blake G.A.: ARA\&A 36, 317 (1998)

24. van Dishoeck E.F., Tielens, A.G.G.M.: to appear in The Century of Space Sciences, eds. J. Bleeker et al. (Kluwer, Dordrecht) in press

25. Whittet D.C.B., Schutte W.A., Tielens A.G.G.M. et al.: A\&A 315, L357 (1996)

26. Whittet D.C.B. et al.: ApJ 498, L159 (1998) 